\title{
First principles calculation of electronic, phonon and thermal properties of hydrogenated germanene
}

\author{
LEI LIU*®, YANJU JI and LIQIANG LIU \\ School of Science, Shandong Jianzhu University, Jinan, Shandong, People's Republic of China \\ *Author for correspondence (2922977166@qq.com)
}

MS received 11 November 2018; accepted 31 December 2018; published online 13 May 2019

\begin{abstract}
Germanene is a basic building block of two-dimensional materials of germanium and it exhibits many unique electronic properties. It is necessary for germanene to tuning its electronic band structure for future applications. The electronic and vibrational properties of germanene, germanane, single-sided semi-hydrogenated germanene and single-sided full-hydrogenated germanene (FHgermanene) were analysed by density function theory. It was found that hydrogenation effectively leads to germanene transition from metallic to semiconductors. Meanwhile, phonon dispersion showed that germanane and FHgermanene are stable. For the same $\mathrm{Ge} / \mathrm{H}$ ratio in the structure, the thermal properties of germanane and FHgermanene are consistent. The hydrogenation process provides a novel method to tune the properties of germanene with unprecedented potentials for future nanoelectronics.
\end{abstract}

Keywords. First principle; germanene; thermal properties; phonon dispersion.

\section{Introduction}

Two-dimensional materials of graphene and graphene analogues, such as silicene, germanene and stanene, have extensively been studied and discussed due to their superior properties [1-6]. Germanene is a single-atom thick sheet of puckered germanium atoms which has been produced and studied theoretically [7-12]. Because it has no band gap and high electron mobility, it is forecasted that germanene can be used in applications of nanodevices [13]. When the electrons in germanene and surface adsorbates interact, it can produce a central issue for modulating the physical properties of germanene. The hydrogenation of graphene analogue materials is a prototype case among these adsorbates as a result of its two-fold interest: not only can it be used to tune the band gap but also it can be harnessed as a hydrogen storage material $[14,15]$. Jose et al [16] theoretically studied the buckling distortions in silicene, which make them excellent materials for band-gap tuning through hydrogenation. All the hydrogenated silicenes have the highest occupied molecular orbital-lowest unoccupied molecular orbital gaps greater than $4 \mathrm{eV}$, indicating a shift from the zero gap to wide gap semiconductors. The increase in the band gap during saturation of silicenes with hydrogens can be used for band gap tuning by controlled saturation by hydrogen. Musin and Wang [17] concluded that with the change in hydrogen concentration on germanene, germanene is transformed from the metal semiconductor to the magnetic semiconductor and then to the non-magnetic semiconductor. Because of the hydrogenated germanene, the chemical surface can be further modified to adjust the band gap, temperature dependent stability, or other properties of the material.

Germanane, full-hydrogenated germanene (FHgermanene), was first generated in an experiment in 2013 by Elisabeth Bianco and others in The Ohio State University. They found that the direct band gap of germanane was $1.53 \mathrm{eV}$ and electron mobility was five times higher than that of bulk germanium [18]. Another possible hydrogenated germanene is single-sided semi-hydrogenated germanene (SHgermanene). Wang et al [12] theoretically demonstrated that the unilateral germanene hydride produces local and unpaired $4 p$ electrons in unhydrogenated germanium atom and then ferromagnetism is introduced into the germanene monolayer without doping, cutting or etching. SHgermanene has direct energy gaps and the value of the band gap obtained from the Perdew-BurkeErnzerhof-generalized gradient approximation functional is $1.32 \mathrm{eV}$.

However, the electronic properties and phonon dispersion of various hydrogenated germanene have not been compared and analysed on theory completely.

In this paper, four structures, germanene, germanane (FHgermanene), SHgermanene and single-side FHgermanene, were studied by first principles calculation. Phonon dispersion was used to examine the stability of structures. The band structure, density of states and some thermal properties were also calculated to analyse the differences between three kinds of hydrogenated germanene. The obtained results can be used to instruct the experiments and practical application of these germanene derivatives. 

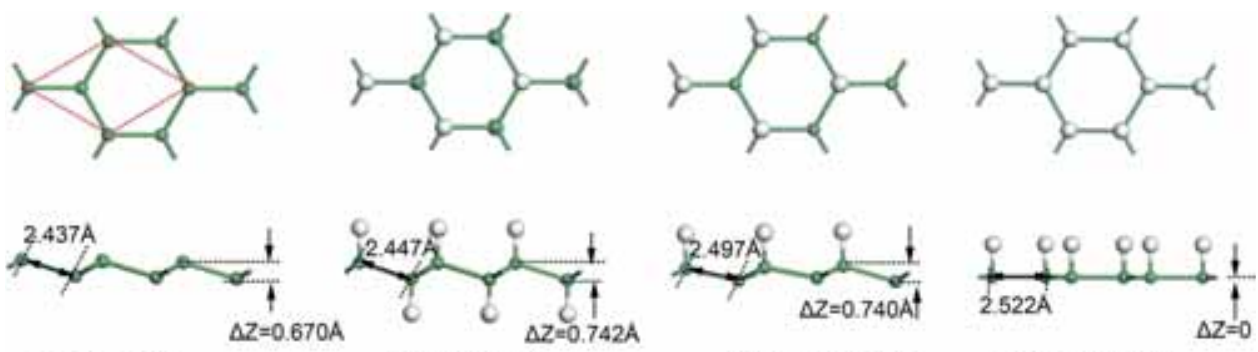

(a)germanene

(b)germanane

(c) SHgermanene

(d)FHgermanene

Figure 1. Side and top views of the ball and stick structures of optimized germanene and hydrogenated germanene: (a) germanene; (b) germanane; (c) SHgermanene and (d) FHgermanene. The green and white spheres denote $\mathrm{Ge}$ and $\mathrm{H}$ atoms, respectively.

\section{Computational method}

In this work, all calculations were performed using the density functional theory with a plane-wave basis set as implemented in the CASTEP code [19]. The core electrons were treated with the norm-conserving pseudopotential. The exchange correlation potential was approximated by the generalized gradient approximation using the PerdewBurke-Ernzerhof function [20,21]. A $750 \mathrm{eV}$ cutoff for the plane-wave basis set was used in all computations. All structures were treated under periodic boundary conditions, and the supercell was large enough to ensure a vacuum spacing greater than $20 \AA$. The valence electron configurations were $4 \mathrm{~s}^{2} 4 \mathrm{p}^{2}$ for $\mathrm{Ge}$ and $1 \mathrm{~s}^{1}$ for $\mathrm{H}$. The Monkhorst-Pack special $k$-point of $9 \times 9 \times 1$ meshes was used to represent the Brillouin zone for all the slab models [22]. The convergence tolerance of energy of $5.0 \times$ $10^{-6} \mathrm{eV}$ per atom, maximum force of $0.01 \mathrm{eV}^{-1}$, maximum displacement of $5.0 \times 10^{-4} \AA$ and maximum stress on the cell of $0.02 \mathrm{GPa}$ were taken in the geometry optimization. The vibrational properties are calculated within the density-functional perturbation theory, which can calculate arbitrarily phonon frequencies without using a lager supercell.

\section{Results and discussion}

\subsection{Crystal structure}

The structures in this study were in the original cell and maintained hexagonal symmetry. The constant, $a$, is the lattice parameter. The pristine germanene and its hydrogenated derivatives used in analysis were fully optimized both the cell geometry and atomic positions. The optimized geometries of four structures are shown in figure 1 .

The calculated lattice constants, bonding parameters and binding energies of the optimized structures are tabulated in table 1 . The pristine germanene is a single-atom-thick monolayer of unsaturated germanium atoms with the hexagonal crystal lattice. The calculated lattice constants and bonding parameters of germanene are in reasonable agreement with the available experimental data [8]. For germanane, every alternate germanium atom is attached to the hydrogen atom from alternate sides of the plane. In response to the additional hydrogen atoms, the germanium atoms are displaced out of the plane towards hydrogen atoms, which leads to the germanium atoms in germanene in a zigzag configuration. The optimized bond lengths of $\mathrm{Ge}-\mathrm{Ge}$ and $\mathrm{Ge}-\mathrm{H}$ are 2.47 and $1.55 \AA$, respectively, which are well consistent with the results of $\mathrm{Li}$ and $\mathrm{Chen}$ [23]. The angle between the two $\mathrm{Ge}-\mathrm{Ge}$ bonds is about $111.41^{\circ}$ while the angle between the $\mathrm{Ge}-\mathrm{Ge}$ and $\mathrm{Ge}-\mathrm{H}$ bonds is about $107.46^{\circ}$. Each germanium atom has four valence electrons and is bonded with four atoms surrounding it, which indicates that germanene is a $\mathrm{sp}^{3}$ hybrid system. For SHgermanene, the relaxed bond length of $\mathrm{Ge}-$ Ge is $2.497 \AA$, which is larger than that in germanene and germanane structures. The enlarging of the lattice constant is due to the increase in the $\mathrm{Ge}-\mathrm{Ge}$ bond length $(2.437 \AA$ for germanene and $2.447 \AA$ for germanane, see table 1 ). The Ge atoms that adsorbed $\mathrm{H}$ atoms in SHgermanene became $\mathrm{sp}^{3}$ hybridized, while other Ge atoms present the $\mathrm{sp}^{2}$ type bond. The optimized FHgermanene is different from that of the former three structures, whose $\mathrm{Ge}$ atoms are in a flat plane. The lattice constant for FHgermanene is $4.367 \AA$, which is larger than that of the former structures. In FHgermanene, all the hydrogen atoms are held at the same side, which naturally results in repulsion between the hydrogen atoms. This further causes the crystal to expand.

The binding energies are calculated as the difference in total energies between the compound and the pseudoatoms for the same setting. The bonding energy is the measure of bond strength in a chemical bond. It is the average of all the bond dissociation energy in the molecule and will show different values for given bond than the bond dissociation energy would. The higher the binding energy, the more stable the structure. Among the four structures, the order of bonding energy is germanane > FHgermanene $>$ germanene $>$ SHgermanene. Germanium atoms of germanene are $\mathrm{sp}^{2}$ hybrid and there are double bonds between two germanium atoms. One of the double bonds belongs to the high-energy $\pi$ bond, which is unstable 
Table 1. The calculated lattice parameters $(a)$, bonding lengths $(d)$, bond angles and binding energies $(\Delta E)$ of germanene and its derivatives.

\begin{tabular}{lcccc}
\hline & Germanene & Germanane & SHgermanene & FHgermanene \\
\hline$a(\AA)$ & 4.058 & 4.092 & 4.117 & 4.367 \\
$d_{\mathrm{Ge}-\mathrm{Ge}}(\AA)$ & 2.437 & 2.447 & 2.497 & 2.522 \\
$d_{\mathrm{Ge}-\mathrm{H}(\AA)}$ & - & 1.560 & 1.583 & 1.565 \\
$\angle \mathrm{Ge}-\mathrm{Ge}-\mathrm{Ge}\left(^{\circ}\right)$ & 112.745 & 111.406 & 111.032 & 120.000 \\
$\angle \mathrm{Ge}-\mathrm{H}-\mathrm{Ge}\left(^{\circ}\right)$ & - & 107.459 & 107.861 & 0 \\
$\Delta Z(\AA)$ & 0.670 & 0.742 & 0.740 & 34.752 \\
$\Delta E$ per atom $(\mathrm{eV})$ & 3.612 & 3.853 & 3.421 & 3.752 \\
\hline
\end{tabular}

(a)
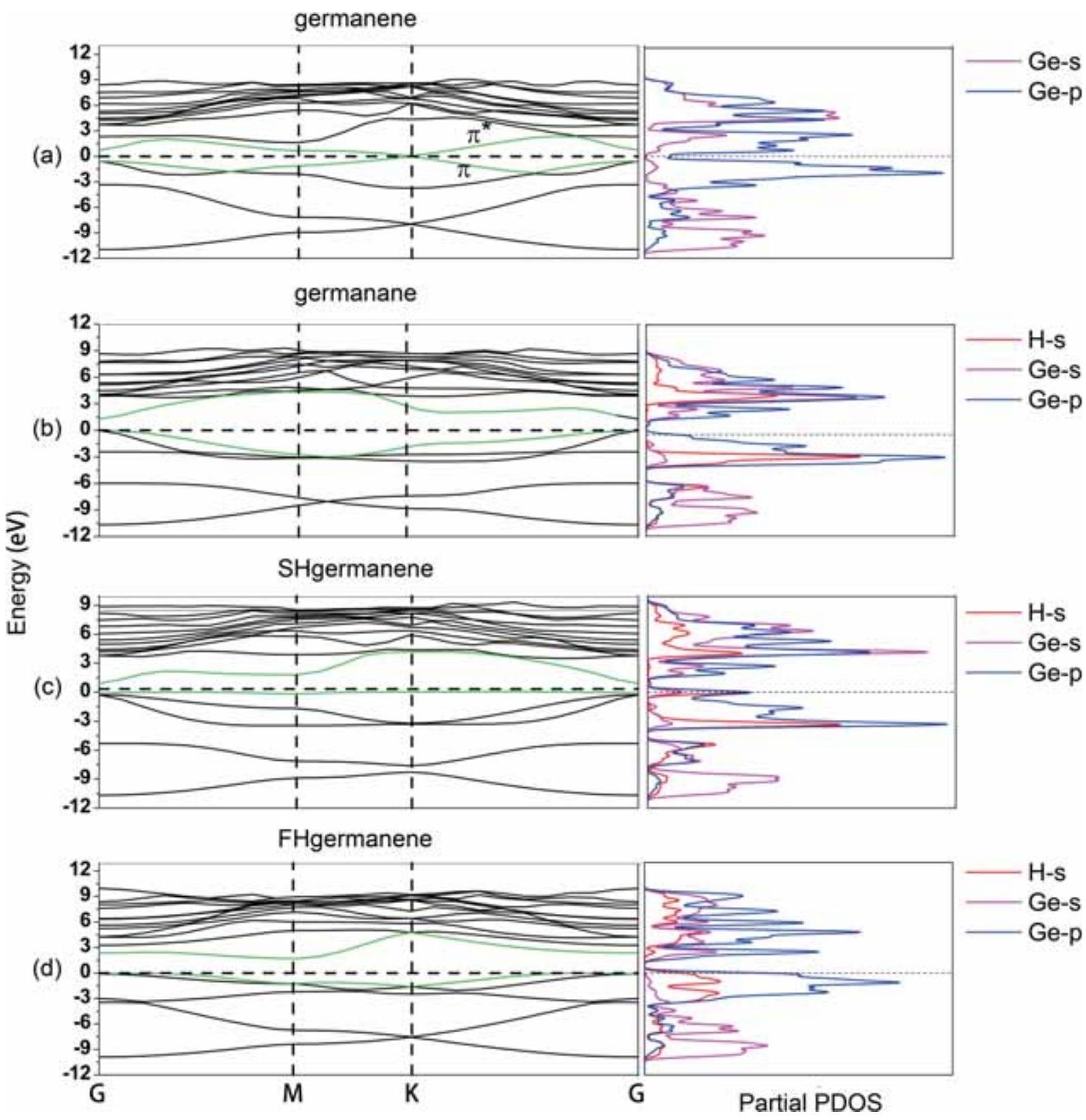

Figure 2. Band structures and electronic density of states of germanene and hydrogenated germanene. 
(a)

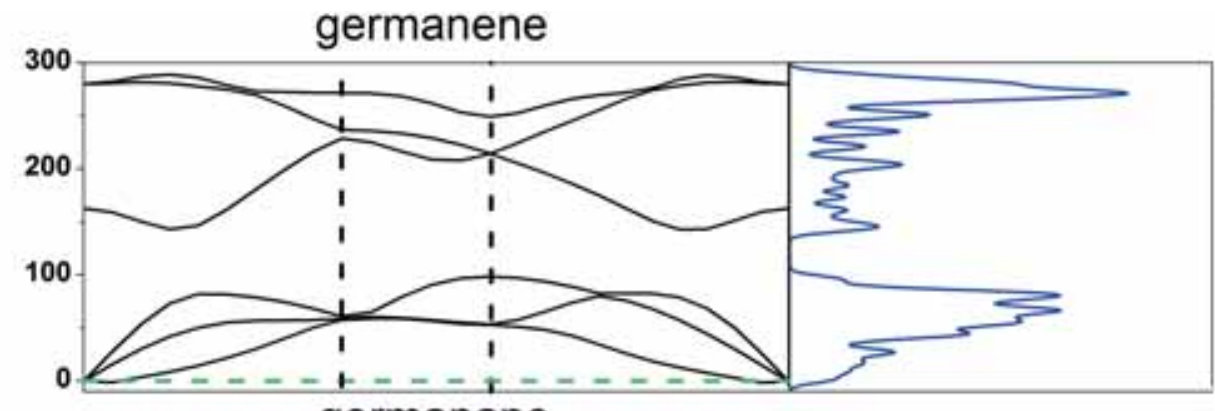

$-\mathrm{Ge}$

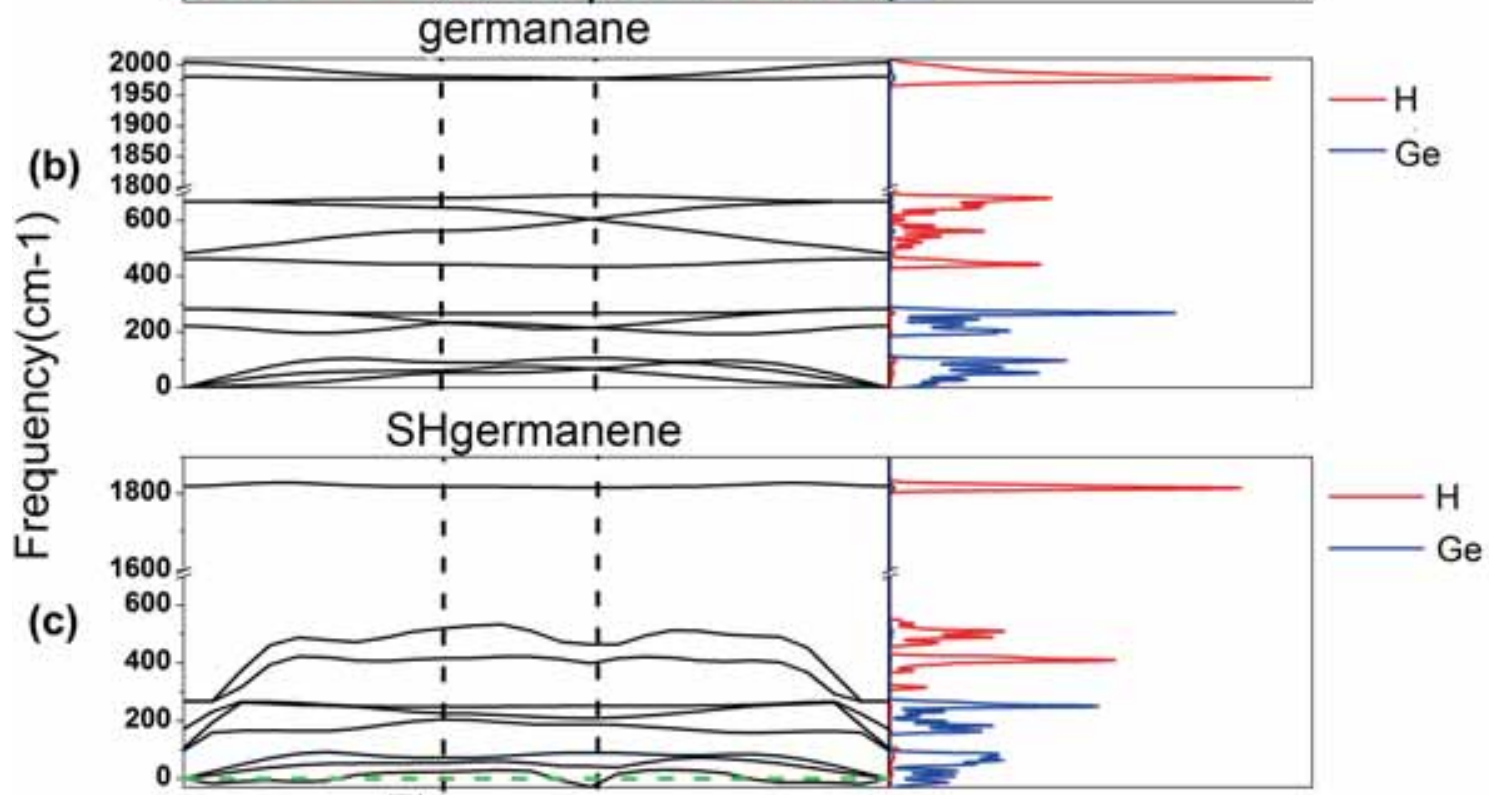

FHgermanene

(d)

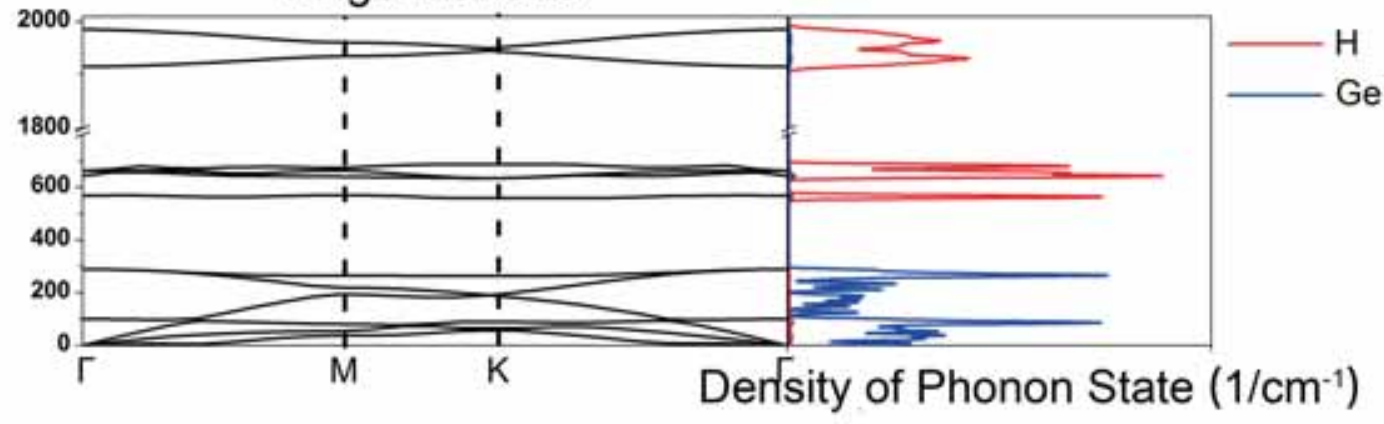

Figure 3. Phonon dispersions and corresponding phonon density of states of germanene and hydrogenated germanene.

and fragile. The addition of hydrogen atoms to germanene destroys unsaturated double bonds, forming more stable hydrocarbon single bonds between hydrogen atoms and original unsaturated bond atoms, which lowers the total energy. So after hydrogenation, germanane and FHgermanene are more stable. The stability can be proved by phonon dispersions without imaginary frequency that are discussed below.

To further assess the stabilities of these germanene derivatives, we also provided the results of the formation energies for various hydrogenated germanene systems. The formation energy is the energy that needs to be absorbed or released by atoms from the elemental state to compounds. The formation energies $E_{\mathrm{f}}$ are calculated as $E_{\mathrm{f}}=E_{\mathrm{H}-\text { germanene }}-E_{\text {germanene }}-$ $n_{\mathrm{H}} E_{\mathrm{H}_{2}} / 2$, where $E_{\mathrm{H} \text {-germanene }}$ and $E_{\text {germanene }}$ are the total energies of hydrogenated and pristine germanene sheets per unit cell respectively, and $E_{\mathrm{H}_{2}}$ is the energy of molecular hydrogen. $n_{\mathrm{H}}$ is the concentration of $\mathrm{H}$ atoms per unit cell. Our calculations show that germanane is the ground state structure with the lowest formation energy of $-2.97 \mathrm{eV}$. The negative value indicates that the hydrogenation is an exothermic reaction. The SHgermanene and FHgermanene conformations are -0.13 and $-1.43 \mathrm{eV}$, both higher in energy compared to germanane. The calculation of formation also showed that germanane and FHgermanene are relatively stable. 


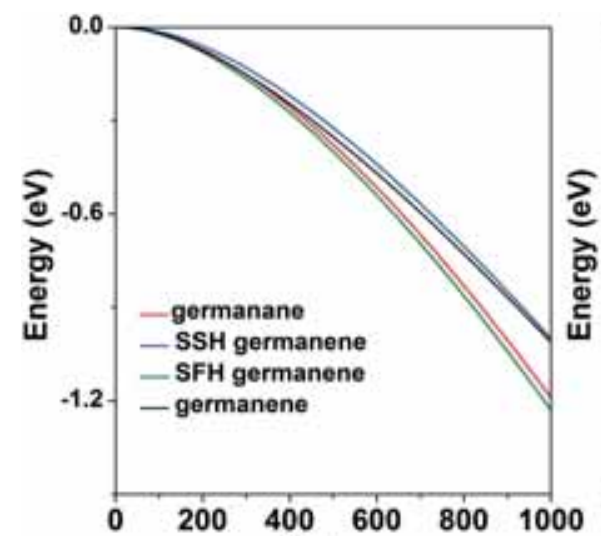

(a)

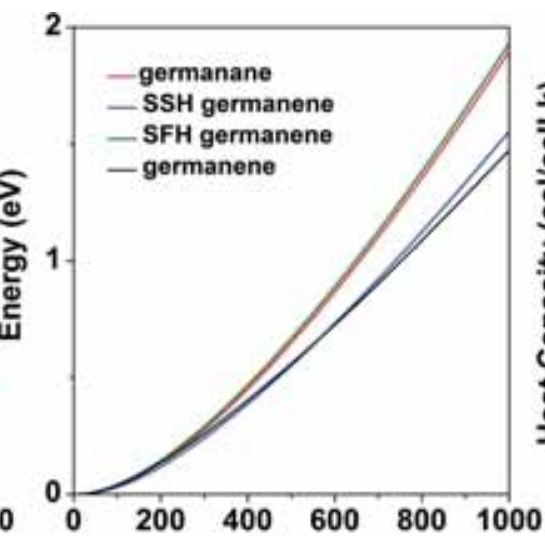

(b)

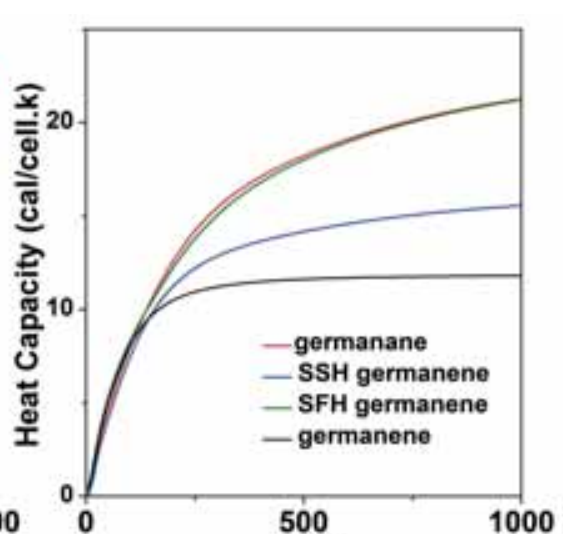

(c)

Temperature(K)

Figure 4. (a) Free energy, (b) vibrational entropy and (c) constant volume specific heat of germanene and its hydrogenated derivatives.

\subsection{Electronic and phonon properties}

It is meaningful to study the hydrogenated derivative of germanene and its electronic structures before discussing their phonon band structures. Note that the literature has reported the electronic structures of hydrogenated germanene [24-27]. But different computational methods were used for different compounds in the previous theoretical work. There was little work where a unified method was employed for all hydrogenated germanene. Here we apply the same method to recalculate all the electronic structural properties of these hydrogenated germanenes.

The calculated results for the electronic band structures and electronic density of states of the hydrogenated germanene as well as with pristine germanene are shown in figure 2 . The bottom of the conduction band and top of the valence band, which are the most important, are marked in green. Figure 2a shows that the valence and conduction bands of germanene are linear and have a zero band gap at $\mathrm{K}$ point. This behaviour of bands attributes a mass-less Dirac fermion character to the charge carriers. This conclusion is in good agreement with Cahangirov's result [28]. The valence and conduction bands are the $\pi$ and $\pi^{*}$ bands, which combined mainly by the perpendicular $\mathrm{p}_{z}$ orbit and little $\mathrm{s}$ orbit.

Figure $2 \mathrm{~b}$ shows that upon adsorption of hydrogen, an energy gap opens at $1.282 \mathrm{eV}$ in germanane. For the introduction of $\mathrm{H}$ atom, a $\mathrm{H}$-s orbit is added to the top of the valence band gap, while the Ge orbit did not change much, which indicated that a bond formed between the H-s and the Ge- $\mathrm{p}_{z}$ mainly contributed to the formation of orbitals. The fourth covalent $\mathrm{Ge}-\mathrm{H}$ bond was different from the former three $\mathrm{Ge}-$ Ge bonds. Therefore the Ge atoms in the germanane were not the typical $\mathrm{sp}^{3}$ hybrid bond. The bottom of the conduction band maintained the hybridization of Ge-p and Ge-s orbits. The formation of the Ge-H bond lowered the energy of the valence band and the band gap opened. Figure $2 \mathrm{c}$ shows that
SHgermanene has a direct band gap value of $0.893 \mathrm{eV}$. The s orbit of the $\mathrm{H}$ atom contributed to the top of the valence band. The band structure of SHgermanene demonstrated that H-s has no bandwidth and its corresponding density of states was sharp. This property was due to only one $\mathrm{H}$ atom at one side and the distance between $\mathrm{H}-\mathrm{H}$ atoms was large, so they had little interaction. Therefore the band of the H-s orbit did not expand. The electronic properties of FHgermanene are shown in figure 2d. The primitive cell of FHgermanene has the same ratio of $\mathrm{Ge} / \mathrm{H}$ as germanane, but all the hydrogen atoms are held at the same side. The bottom of the conduction band shifts upward in energy, which gives rise to the indirect band gap of $1.669 \mathrm{eV}$ between the $\Gamma$ and $\mathrm{M}$ points. The top of the valence band was still mainly contributed by Ge-p and H-s orbits, but the H-s orbit was not sharp anymore and had large bandwidth. The peculiar band structure is due to the enhanced repulsion between the hydrogen atoms that result in the shift of the most sensitive $\mathrm{p}_{z}$-type band of the system. The analysis of individual bands shows that there is a $\sigma$ bond established between the $\mathrm{p}_{z}$ orbital of $\mathrm{Ge}$ and s orbitals of hydrogen, and also indicates a substantial s-s overlap between the two neighbouring hydrogen atoms causing repulsion. The conclusion shows that due to the nature of orbitals, FHgermanene is not a strict $\mathrm{sp}^{3}$-bonded system.

Figure 3 shows the calculated phonon dispersions for hydrogenated germanene. For the purpose of comparison, the phonon band structure of pristine germanene is also presented. The primitive cell of the germanene monolayer has two atoms, suggesting that the spectrum should have 6 lines. These lines will degenerate, as shown in figure $3 \mathrm{a}$, at the high symmetry $\Gamma$ point. The transverse acoustical phonon branch has imaginary frequencies near the point. This interpreted that the structure of germanene is unstable. The primitive cell of the germanane monolayer has four atoms, suggesting that the spectrum should have 12 lines. As can be seen in figure $3 b$, the absence of imaginary frequency indicates that 
germanane is stable. It is clear that the phonon bands of germanane can be divided into distinct low-, intermediate- and high-frequency part phonons. The right hand-sided panels in figure $3 \mathrm{~b}$ present the corresponding phonon density of states (PDOSs). The blue and red curves represent the PDOSs projected onto the $\mathrm{Ge}$ and $\mathrm{H}$ atoms, respectively. It is shown that the both high-frequency and intermediate-frequency phonons in germanane are dominated by $\mathrm{H}$ models. The low-frequency acoustic phonons are due to Ge models in hydrogenated germanene. It was worth noting that there were longitudinal optical (LO)/transversal optical (TO) splitting lines in the frequency range of $200-500 \mathrm{~cm}^{-1}$ at the $\Gamma$ point, while the frequency near $700 \mathrm{~cm}^{-1}$ did not appear the LO/TO splitting. In the structure of germanane the long range of the Coulomb interactions should lead to the frequencies of LO modes above those of TO modes. Figure $3 \mathrm{c}$ shows the phonon properties for SHgermanene. It appears that some are imaginary modes and the system is unstable. Figure $3 \mathrm{~d}$ shows the phonon properties of FHgermanene. For FHgermanene, the intermediate-frequency phonons and high-frequency are also mainly contributed by $\mathrm{H}$ atom, but the peak of the intermediate is higher than that of high-frequency while germanane is on the contrary. These changes are due to the enhanced interaction between the $\mathrm{H}$-s orbit and $\mathrm{H}$-s orbit.

\subsection{Thermal properties}

Based on the obtained phonon density of states, we further calculated the free energy, entropy and constant volume specific heat of the hydrogenated germanene in terms of the harmonic approximation within the following equations [29]:

$$
\begin{aligned}
F(T)= & 3 k_{\mathrm{B}} T \int_{0}^{\omega_{\mathrm{L}}} \ln \left[2 \sinh \frac{\hbar \omega}{k_{\mathrm{B}} T}\right] g(\omega) \mathrm{d} \omega \\
S(T)= & 3 k_{\mathrm{B}} \int_{0}^{\omega_{\mathrm{L}}}\left[\frac{\hbar \omega}{2 k_{\mathrm{B}} T} \operatorname{coth} \frac{\hbar \omega}{2 k_{\mathrm{B}} T}\right. \\
& \left.-\ln \left(2 \sinh \frac{\hbar \omega}{k_{\mathrm{B}} T}\right)\right] g(\omega) \mathrm{d} \omega \\
\mathrm{C}_{\mathrm{V}}(T)= & 3 k_{\mathrm{B}} \int_{0}^{\omega_{\mathrm{L}}}\left(\frac{\hbar \omega}{2 k_{\mathrm{B}} T}\right)^{2} \csc h^{2}\left(\frac{\hbar \omega}{k_{\mathrm{B}} T}\right) g(\omega) \mathrm{d} \omega
\end{aligned}
$$

where $\omega_{\mathrm{L}}$ is the largest phonon frequency, $g(\omega)$ is the phonon density of states normalized to unit, $k_{\mathrm{B}}$ is the Boltzmann constant and $T$ is the temperature.

The results for free energy, entropy and heat capacity as a function of temperature are shown in figure 4 . The calculated thermodynamics properties which are shown in figure are limited to $1000 \mathrm{~K}$. Figure 4 shows that there are significant differences in them for different hydrogenated germanenes. Germanene and FHgermanene have the same Ge/H ratio, the temperature dependencies of free energy, entropy and heat capacity are similar. Germanene is found to be having lower entropy than its hydrogenated derivatives over the whole temperature range as shown in figure $4 c$, due to its absence of phonon density of states for intermediate-frequency and highfrequency modes, as shown in figure 3 . The classic limit of the heat capacity can be expected by the Dulong-Perit law and is equal to $3 R / M$, where $R$ is the ideal gas constant and $M$ is the molar mass. Because the molar mass of germanane is the same as that of FHgermanene, the value of the heat capacity will be equal at high temperature, as shown in figure $4 c$.

\section{Conclusions}

In summary, we have investigated the structural, electronic and vibrational properties of germanene and hydrogenated germanene (germanane, SHgermanene and FHgermanene) by means of first-principles calculations. The four structures maintained hexagonal symmetry and FHgermanene has the largest lattice parameter.

The calculation showed that germanane and FHgermanene are more executable. The analysis of bonding energy indicated that they were energetically stable. The phonon dispersion demonstrated that they were structurally stable. The two stable germanene derivatives, germanane and FHgermanene, have a direct gap of $1.28 \mathrm{eV}$ and an indirect gap of $1.699 \mathrm{eV}$, respectively. Both germanane and FHgermanene, with the same $\mathrm{Ge} / \mathrm{H}$ ratio, showed similar heat capacity. The research of germanene, germanane, SHgermanene and FHgermanene is useful for developing optimized strategies to characterize the evolution of functionalized germanene.

\section{References}

[1] Zhang Y, Tan Y W, Stormer H L and Kim P 2005 Nature 438 201

[2] Lee C, Wei X, Kysar J W and Hone J 2008 Science 321385

[3] Le Lay G 2015 Nat. Nanotechnol. 10202

[4] Jose D and Datta A 2014 Acc. Chem. Res. 47593

[5] Jose D and Datta A 2012 J. Phys. Chem. C 11624639

[6] Ghosh M and Datta A 2018 Bull. Mater. Sci. 41117

[7] Ni Z, Liu Q, Tang K, Zheng J, Zhou J, Qin R et al 2011 Nano Lett. 12113

[8] Dávila M E, Xian L, Cahangirov S, Rubio A and Le Lay G 2014 New J. Phys. 16095002

[9] Balendhran S, Walia S, Nili H, Sriram S and Bhaskaran M 2015 Small 11640

[10] Mandal T K, Jose D, Nijamudheen A and Datta A 2014 J. Phys. Chem. C 11812115

[11] Nijamudheen A, Bhattacharjee R, Choudhury S and Datta A 2015 J. Phys. Chem. C 1193802

[12] Wang Y, Zheng J, Ni Z, Fei R, Liu Q, Quhe R et al 2012 Nano 7 1250037

[13] Houssa M, Scalise E, Sankaran K, Pourtois G, Afanas' Ev V V and Stesmans A 2011 Appl. Phys. Lett. 98223107

[14] Dekura S, Kobayashi H, Ikeda R, Maesato M, Yoshino H, Ohba M et al 2018 Angew. Chem. Int. Ed. 579823 
[15] Seixas L, Padilha J E and Fazzio A 2014 Phys. Rev. B 89 195403

[16] Jose D, Chowdhury C and Datta A 2018 in: P Vogt and G Le Lay (eds) A vision on organosilicon chemistry and silicene (Silicene. NanoScience and Technology, Springer, Cham), p 1

[17] Musin R N and Wang X Q 2006 Phys. Rev. B 74165308

[18] Bianco E, Butler S, Jiang S, Restrepo O D, Windl W and Goldberger J E 2013 ACS Nano 74414

[19] Segall M D, Lindan P J, Probert M A, Pickard C J, Hasnip P J, Clark S J et al 2002 J. Phys. Condens. Matter 142717

[20] Schwarz K, Blaha P and Madsen G K 2002 Comput. Phys. Commun. 14771

[21] Fischer T H and Almlof J 1992 J. Phys. Chem. C 969768
[22] Chadi D J 1977 Phys. Rev. B 135188

[23] Li Y F and Chen Z 2014 J. Phys. Chem. C 1181148

[24] Wei W, Dai Y, Huang B and Jacob T 2013 Phys. Chem. Chem. Phys. 158789

[25] Rojas K I M, Al Rey C V, Moreno J L, David M and Arboleda Jr N B 2018 Int. J. Hydrogen Energy 434393

[26] Hattori A, Tanaya S, Yada K, Araidai M, Sato M, Hatsugai Y et al 2017 J. Phys. Condens. Matter 29115302

[27] Monshi M M, Aghaei S M and Calizo I 2017 RSC Adv. 718900

[28] Cahangirov S, Topsakal M, Aktürk E, Şahin H and Ciraci S 2009 Phys. Rev. Lett. 102236804

[29] Perdew J P, Burke K and Ernzerhof M 1998 Phys. Rev. Lett. 80 891 